\title{
Design and Identification of Two-Dimensional Code Anti-Distortion on Cylinder Surface Based on Pre-Stretching
}

\author{
You Fu Cheng ${ }^{1}$, Jiang Chao ${ }^{2}$, Zhang Dong ${ }^{3}$, Li Ming ${ }^{4}$, Cao Yue ${ }^{5}$ \\ and Zhang Chen Wei ${ }^{6}$ \\ 1, 2, 3,4,5,6 Beijing Institute of Graphic Communication \\ 1youfucheng@bigc.edu.cn, ${ }^{2} 13167333633 @ 163 . c o m$, \\ ${ }^{3}$ Email:zd18610221717@sina.com, ${ }^{4} 18010096220 @ 163 . c o m$, \\ ${ }^{5}$ caoyueroy@aliyun.com, ${ }^{6}$ weixiansuiyue@sina.cn
}

\begin{abstract}
In order to ensure to be correctly identified by the acquisition devices, the traditional two-dimensional code must be attached to the surface plane of the object. If attached to the surface of the cylinder, the image of the normal two-dimensional code will be compressed into distortion because of image projection, which will lead to the problem that the image acquisition device cannot identify it. Aiming at this problem, a method of design and identification of anti-distortion two-dimensional code on cylindrical surface based on pre-stretching is proposed. According to the spatial geometric relation of the two -dimensional code carrier, in the light of the requirement of conformal projection inverse mapping, the normal two-dimensional code pictures are preliminarily and nonlinearly stretched to obtain the pre-deformed two-dimensional code that will be printed and then pasted on the surface of the cylinder. At this point, the image of the pre-stretched two-dimensional code in the projection plane of the acquisition device is just as the normal two-dimensional code, which will be correctly identified. Finally, the effectiveness of the method is proved by experimental simulation.
\end{abstract}

Keywords: Cylinder surface; pre-stretching; two-dimensional code; Projection transformation

\section{Introduction}

With the rapid development of wireless network technology and the rapid spread of mobile intelligent devices, two-dimensional code technology impact on people's way of life as a "bridge" of information exchange profound, bringing huge social and economic benefits. Two-dimensional code technology is an important part of perceptual recognition technology. As a kind of information storage and transmission technology, two-dimensional code is a planar geometric application formed according to the specific coding rules[1-2]. It can express information in both longitudinal and transverse directions, and has the characteristics of large storage capacity, high confidentiality, high tracking ability, strong resistance to damage, high redundancy and low cost and other characteristics[3]. The two-dimensional code expands the encoding capacity, and the scope of the code expanded to the number, letters, Chinese, pictures and sound. What's more, it can quickly correct the error code word, and the process of use of two-dimensional code could be independent of the database[4]. The application of two-dimensional code is becoming more and more abundant[5], involving business management, food safety, advertising marketing, taxation, tourism and other fields. However, the position of the carrier of the current two-dimensional code is limited, and it

Received (April 14, 2017), Review Result (July 10, 2017), Accepted (July 20, 2017) 
is necessary to print the two-dimensional code on a nearly flat carrier or flat terminal display screen. Once placed on the surface will make the two-dimensional code image distortion and cannot be identified. This paper presents a scheme for placing a large format two-dimensional code (In this paper, Overlooking the projection arc of two dimensional code on a cylinder $\pi / 2 \sim \pi$ called large-format two-dimensional code)on the surface of the cylinder which does not change the current scan code software to ensure the success of the scan code recognition. Through the spatial geometric, print the pre-stretching two-dimensional code image on the carrier. When the pre-stretching two-dimensional code is placed on the surface of the cylinder, the image of the projection plane is just an undistorted two-dimensional code when look from its center. Any decoding software can scan and decode at the center of the two-dimensional code. This will improve the scope of application of two-dimensional code, so that it can adapt to a large number of drugs, cosmetics, food and other applications of cylindrical surface packaging.

\section{Design Principles and Content}

In daily life, the two-dimensional code we have seen is usually printed or displayed at the approximate plane of the carrier. The two-dimensional code image we collect through the image acquisition device is a flat two-dimensional code image, and it can be decoded by the current two-dimensional code decoding device. However, when the carrier is a cylinder, the two-dimensional code image we collect through the image acquisition device will occur nonlinear deformation. If the two-dimensional code is small compared with the cylinder surface, the two-dimensional code image can still be decoded by decoding software. But in other cases, the large deformation on both sides of the two-dimensional code makes the two-dimensional code can not be decoded by decoding software. Then the existing decoding software needs to be redesigned to decode it. According to the problem described above, a method of design and identification of anti-distortion two-dimensional code on cylindrical surface based on pre-stretching is proposed. The pre-streching is a kind of non-linear stretching which based on the relationship between the shape of two-dimensional code carrier surface and the two-dimensional code image. Put the deformed two-dimensional code image on the surface of the carrier. Then, the current two-dimensional code image is collected by the image acquisition device. Because the effect of projection compression counteracts the effect of pre-stretching, the projection plane is almost the same as the normal two-dimensional code. Because the pre-stretching is start at the center line of the normal two-dimensional code, the deformed two-dimensional code is centrosymmetric. When the image is collected, the image acquisition device need to be placed at the center of the two-dimensional code. Flow chart of specific design is shown in Figure 1, and the three-dimensional map of the cylindrical carrier is shown in Figure 2. 


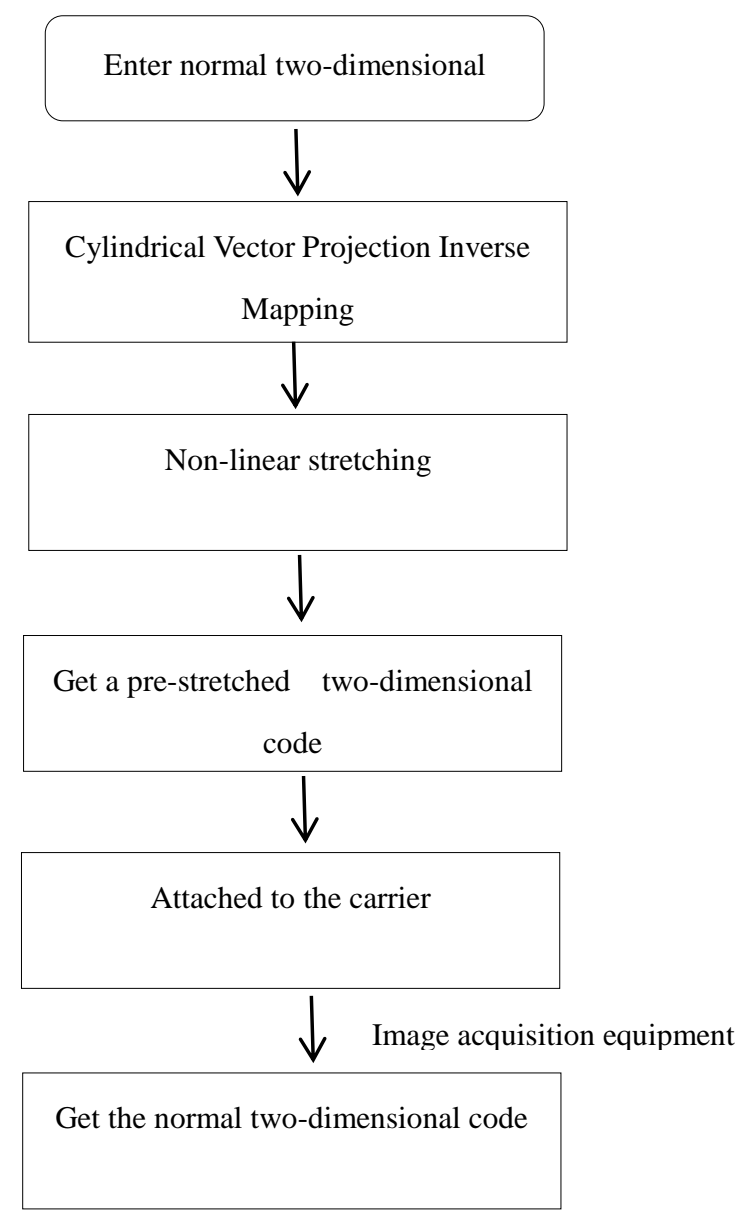

Figure 1. Flow Chart of Specific Design

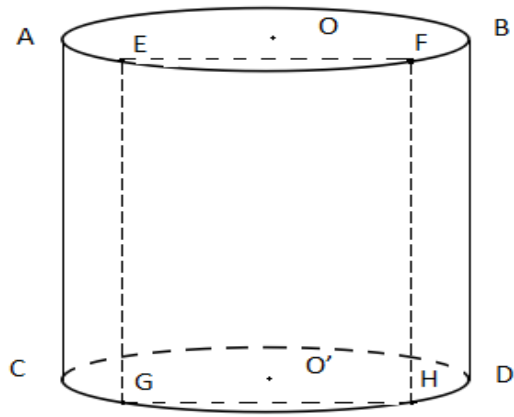

\section{Figure 2. Partial Perspective of a Cylindrical Carrier}

\subsection{Geometry Relation of Cylindrical Vector Projection}

According to the mapping relationship between the two-dimensional code carrier and the two-dimensional code image, the height of the two-dimensional code does not change at the pre-stretching, that is, the image does not produce telescopic deformation in the longitudinal direction. Therefore, it is only necessary to consider a longitudinal carrier of equal height to the two-dimensional code image. As shown in Figure 3, the carrier from $O$ to $O$ ' height is cut into thin slices with unit thickness of pixel, the number of sheets is h (in pixels).Therefore, the carrier can be composed of $\mathrm{H}$ graphics shown in Figure 4. 
Where:

$$
\sum_{i=0}^{h} \pi R^{2} \cdot 1=V
$$

Thus, the line pixel calculation is performed on each circle sheet according to the mapping relationship between the row pixels of the two-dimensional code and the relevant row of the cylinder surface. Then the whole two-dimensional code image is traversed, and the two-dimensional code image is calculated.

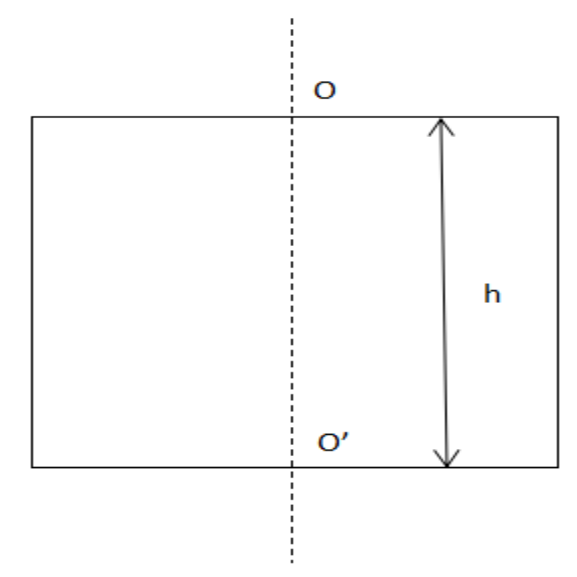

Figure 3. Front View of a Cylindrical Carrier

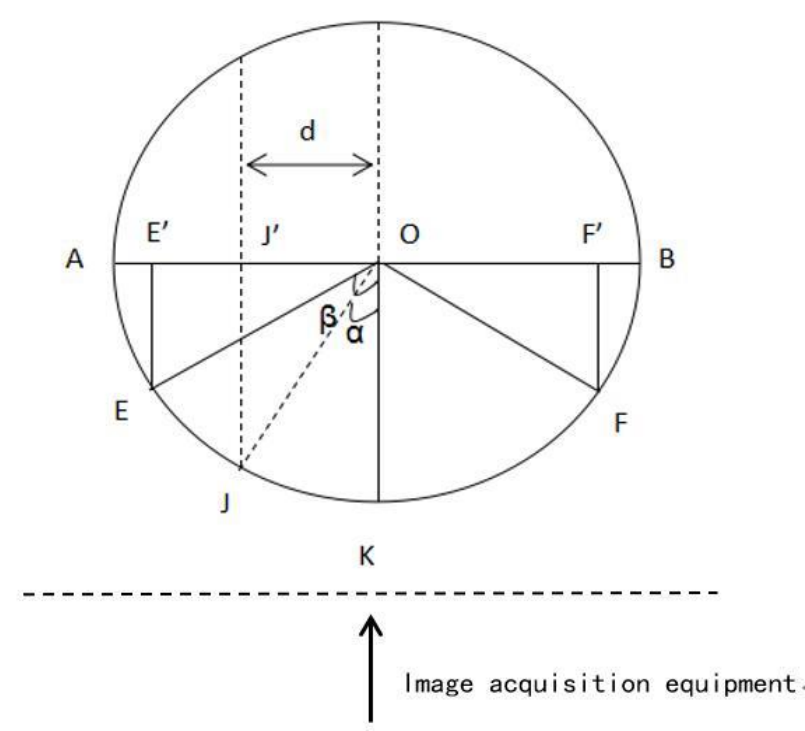

Figure 4. Cross Section of the Cylinder

As shown in Figure $4, l_{E^{\prime} F}$ is the width of the two-dimensional code. In other word, $l_{E^{\prime} F^{\prime}}$ can be used to represent the row pixels of the two-dimensional code image. According to the relational mapping, $\mathrm{EF}$ are the row of pixels corresponding to $l_{E^{\prime} F^{\prime}}$ on the cylindrical carrier, thus, EF represents the row pixels of the deformed two-dimensional code image. According to the above principle, the deformed image can be obtained by following four steps:

Step 1: Find the geometric center of the two-dimensional code image (the symmetry 
axis of the two-dimensional code) which is located at the center of the cylinder;

Step 2: Based on the known size of the two-dimensional code and the diameter of the cylinder, the length of EF can calculated. Then the size of the deformed two-dimensional code is determined. The cylinder is sliced at intervals of one pixel, then take out a cross section of the cylinder for the next step. Calculated as follows:

$$
\begin{gathered}
l_{E^{\prime} O}=l_{E^{\prime} F^{\prime}} / 2 \\
\beta=\arcsin \left(l_{E^{\prime} O} / R\right) \\
l_{E K}=R \square \beta, l_{E F}=2 l_{E K}
\end{gathered}
$$

Step 3: Let point $\mathrm{J}$ is any point on $\mathrm{EF}$, mapped point $\mathrm{J}$ is the point where the moving point $\mathbf{J}$ maps to a line pixel in the two-dimensional code image. The length of EJ mapped on the two-dimensional code image is the distance from mapped point $J$ ' to mapped point E' of two-dimensional code on the left edge.

When point $\mathrm{J}$ moves on the left side of point $\mathrm{K}$, the arc length of $l_{\mathrm{EJ}}$, the angle $\alpha$ (in radians) and the length of $l_{E^{\prime} J}$ relationship are:

$$
\begin{gathered}
l_{\mathrm{JK}}=l_{\mathrm{EK}}-l_{\mathrm{EJ}} \\
\alpha=l_{\mathrm{JK}} / \mathrm{R} \quad(\mathrm{R} \text { radius of circle }) \\
l_{J^{\prime} O}=R \cdot \sin \alpha=d \\
l_{E^{\prime} J^{\prime}}=l_{E^{\prime} O}-l_{J^{\prime} O}
\end{gathered}
$$

When point $\mathrm{J}$ moves on the right side of point $\mathrm{K}$, the arc length of $l_{\mathrm{EJ}}$, the angle $\alpha$ (in radians) and the length of $l_{E^{\prime} J^{\prime}}$ relationship are:

$$
\begin{gathered}
l_{\mathrm{JK}}=l_{\mathrm{EJ}}-l_{\mathrm{EK}} \\
\alpha=l_{\mathrm{JK}} / R \quad(\mathrm{R} \text { radius of circle }) \\
l_{J^{\prime} O}=R \cdot \sin \alpha \\
l_{E^{\prime} J^{\prime}}=l_{E^{\prime} O}+l_{J^{\prime} O}
\end{gathered}
$$

Thus, the mapping arc of the row pixel $E^{\prime} J^{\prime}$ can be obtained.

From the relationship between the arc length and the center angle, we get mapped point $\mathrm{J}$ ' of the normal two-dimensional code only by obtaining the mapped length of $l_{\mathrm{EJ}}$ when point $\mathrm{J}$ moves from the center point $\mathrm{K}$ to the left along the direction of $\mathrm{KE}$.

Step 4: Through mapping from left to right in the normal two-dimensional code row-by-row, we can get unfold drawings of pre-stretched two-dimensional code when traversing the entire two-dimensional code image.

\subsection{The Process of Pre-stretching}

Taking the example that height and width of the QR Code are equal to the diameter of the cylinder. First of all, the normal QR Code image (shown in Figure 5) is read and we get the number of rows : rows $=m$, the number of cols $: c o l s=m$. Because the height and the width of the QR Code are equal to the diameter of the cylinder, the number of the pre-stretched $\mathrm{QR}$ Code of rows is: defRows $=$ rows ( value $\mathrm{h}$ in Figure 3), the number of cols: $\operatorname{defCols}=\operatorname{cols} \cdot \pi / 2$. The following four steps are performed according to Figure 6: 


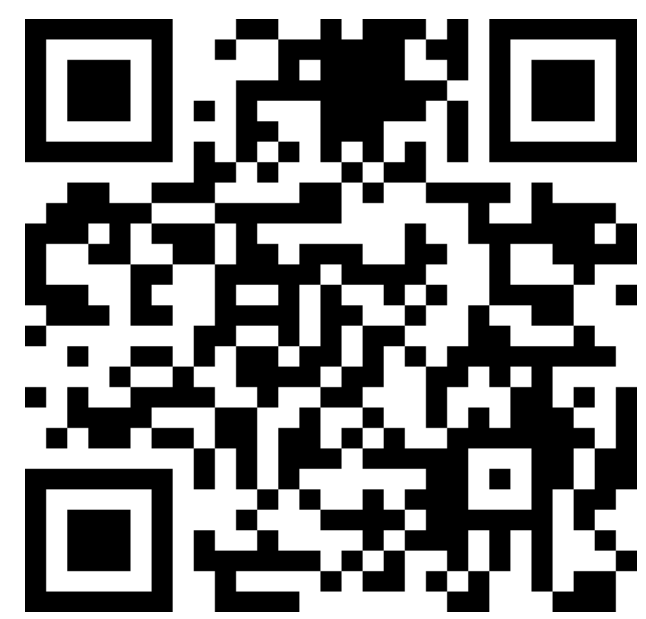

Figure 5. The Normal QR Code

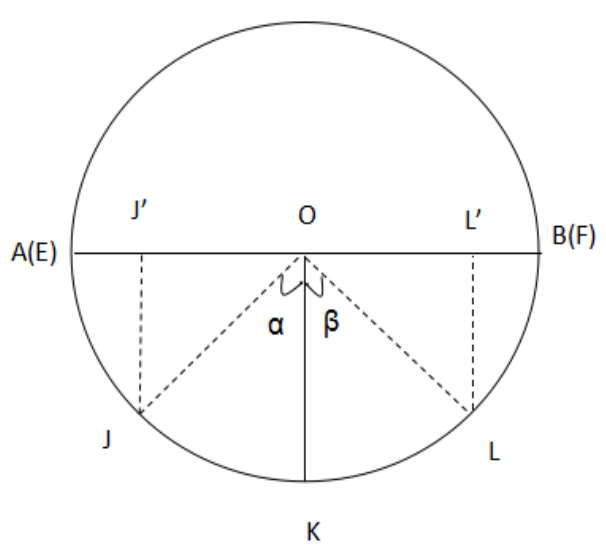

Figure 6. Cross Section of an Example Cylinder

Step 1: The axis position of the QR Code image is the center of the circle $\mathrm{O}, l_{A B}$ is the row of pixels (cylinder diameter) in the QR Code, which is:

$$
\begin{gathered}
l_{A O}=l_{O B}=\mathrm{cols} / 2 \\
l_{A B}=2 l_{A O}
\end{gathered}
$$

As shown in Figure 6, the corresponding point of the center $\mathrm{O}$ is point K. So the pixel value of the center $\mathrm{O}$ is assigned to point $\mathrm{K}$ of the corresponding line.

$$
l_{\mathrm{AK}}=l_{K B}=\text { defCols } / 2
$$

Point $\mathrm{K}$ is the center of a row of pixels in the pre-stretched QR Code. $l_{A B}$ is the number of pixels in the pre-stretched QR Code, where:

$$
l_{A B}=\text { defCols }=\text { cols } \cdot \pi / 2
$$

Step 2: Point $\mathrm{K}$ is the start point which is the midpoint of the line of the pre-stretched two-dimensional code. Point $\mathrm{A}$ is the end point that is the left edge pixel of the pre-stretched QR Code. With point K moving along the $l_{A K}$ to the left edge, the total number of moving pixels is defCols / 2 , and point $\mathrm{J}$ is the moving point in the above process, where: 


$$
l_{\mathrm{AJ}}=\mathrm{m} \quad(0 \leq m<\operatorname{defCols} / 2)
$$

The position of mapped point $\mathrm{J}$ 'on the straight line $l_{A O}$ is calculated from moving point $\mathrm{J}$ on the straight line $l_{A K}$ by equation (1), (2) and (3). So the value of $l_{A J}$, is obtained. We calculate the $n$-th pixel of the row pixel in the pre-stretched QR Code from the m-th pixel of the corresponding row pixel in the normal QR Code, and assign the value of the m-th pixel to the $\mathrm{n}$-th pixel in the pre-stretched QR Code(the above two points $\mathrm{m}, \mathrm{n}$ start counting from the left edge of image).

$$
\begin{gathered}
\alpha=\left(l_{A K}-l_{\mathrm{AJ}}\right) / l_{A O} \\
l_{J^{\prime} O}=l_{J O} \cdot \sin \alpha \\
l_{A J^{\prime}}=l_{A O}-l_{J^{\prime} O}
\end{gathered}
$$

In the equation (1) $\alpha=\angle \mathrm{J}^{\prime} \mathrm{JO}=\square \mathrm{IOK}$ (Radian system), because the point $\mathrm{K}$ is the midpoint of $l_{A B}, l_{A B}=$ defCols is the number of pixels in the pre-stretched QR Code, so $l_{\mathrm{AK}}=l_{\mathrm{AB}} / 2=\operatorname{defCols} / 2$ is the number of a row of pixels in the pre-stretched QR Code. $l_{\mathrm{AJ}}$ represents the distance from the moving point $\mathrm{J}$ to the left edge of the pre-stretched QR Code. $l_{A O}$ represents half the number of pixels of the normal QR Code image, also represent the radius of the cylinder. In the equation (2), $l_{\mathrm{J}^{\prime} O}$ represents the distance from the moving point J' on the $l_{A O}$ to the axis position (the center $\mathrm{O}$ )of the QR Code image. $l_{J O}$ represents the radius of the round sheet, also represents half the number of rows pixels in the normal QR Code image: $l_{J O}=c o l s / 2$.angle $\alpha$ is the value of $\angle \mathrm{J}$ 'JO which calculated in equation (1).In equation (3), $l_{\mathrm{AJ}}$ represents the distance from the moving point J' on the $l_{A O}$ to the position of the left edge of the QR Code image( point A). In this equation, $l_{A O}$ represents the length of the left half of the normal QR Code, and also represents the radius of the circle: $l_{A O}=$ cols $/ 2 . l_{\mathrm{J}^{\prime} O}$ represents the distance from the moving point J' on the $l_{A O}$ to the axis position (the center O)of the QR Code image in equation (2).

Step 3: Point $\mathrm{K}$ is the start point that is the midpoint of the line of the pre-stretched QR Code. Point B is the end point that is the left edge pixel of the pre-stretched QR Code. Point $\mathrm{K}$ moving along the $l_{\mathrm{KB}}$ to the left edge. The total number of moving pixels is defCols / 2 , and point $\mathrm{L}$ is the moving point in the above process.

$$
l_{\mathrm{AL}}=\mathrm{p} \quad(\text { defCols } / 2<p \leq \text { defCols })
$$

The position of mapped point $\mathrm{L}$ 'on the straight line $l_{O B}$ is calculated from moving point L on the straight line $l_{A L^{\prime}}$ by equation (4), (5) and (6). So the value of $l_{A L^{\prime}}$ is obtained. We calculate the p-th pixel of the row pixel in the pre-stretched QR Code from the q-th pixel of the corresponding row pixel in the normal QR Code, and assign the value of the q-th pixel to the $\mathrm{p}$-th pixel in the pre-stretched $\mathrm{QR}$ Code(the above two points $\mathrm{p}, \mathrm{q}$ start counting from the left edge of image).

$$
\begin{gathered}
\beta=\left(l_{A L}-l_{\mathrm{AK}}\right) / l_{\mathrm{OB}} \\
l_{L^{\prime} O}=l_{L O} \cdot \sin \beta
\end{gathered}
$$




$$
l_{A L^{\prime}}=l_{A O}+l_{O L^{\prime}}
$$

In the equation (4) $\beta=\angle O L L^{\prime}=\square K O L$ (Radian system), because the point $\mathrm{K}$ is the midpoint of $l_{A B}$, and $l_{A B}=$ defCols is the number of pixels in the pre-stretched QR Code, so $l_{\mathrm{AK}}=l_{\mathrm{AB}} / 2=\operatorname{defCols} / 2$ is the number of a row of pixels in the pre-stretched QR Code. $l_{\mathrm{AL}}$ represents the distance from the moving point $\mathrm{L}$ to the left edge of the pre-stretched QR Code. $l_{O B}$ represents half the number of pixels of the normal QR Code image, also represent the radius of the cylinder. In the equation (5), $l_{L^{\prime} O}$ represents the distance from the moving point L' on the $l_{O B}$ to the axis position (the center O)of the QR Code image. $l_{L O}$ represents the radius of the round sheet, also represents half the number of rows pixels in the normal QR Code image: $l_{L O}=$ cols $/ 2$. The angle $\beta$ is the value of $\angle O L L^{\prime}$ calculated in equation (4). In equation (6), $l_{A L}$, represents the distance from the moving point L' on the $l_{O B}$ to the position of the left edge of the QR Code image( point A). In this equation, $l_{A O}$ represents the length of the left half of the normal QR Code, and also represents the radius of the circle: $l_{A O}=\operatorname{cols} / 2 . l_{O L}$ represents the distance from the moving point L' on the $l_{\mathrm{KB}}$ to the axis position (the center $\mathrm{O}$ )of the QR Code image in equation (5).

Step 4:Because the diameter of the cylinder is constant, the number of per column pixels is unchanged after the deformation of the normal QR Code. Then we calculate line by line starting at the first line of pixels and end at last line. Through the three steps above, we can traverse the entire QR Code. Then we get the pre-stretched QR Code as shown in Figure 7.

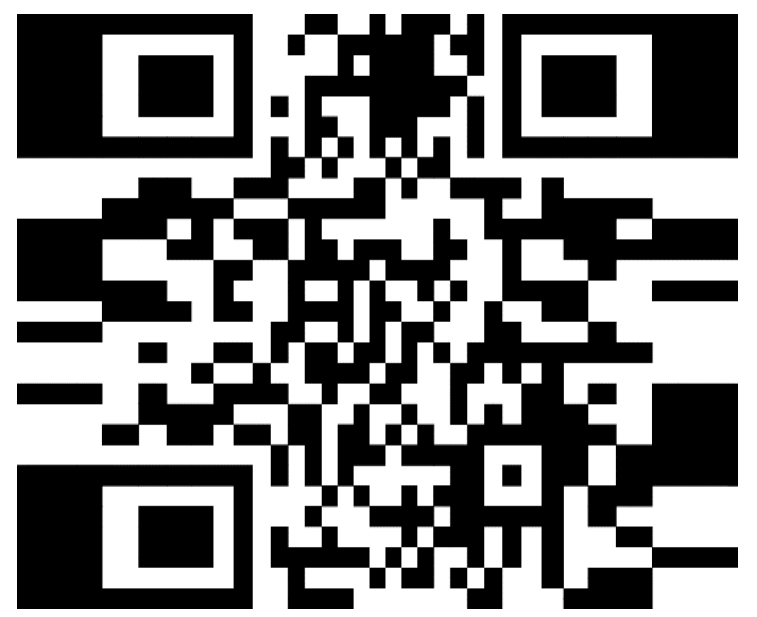

Figure 7. The Pre-stretched QR Code

We print the pre-stretched QR Code to the cylinder side surface. The renderings is collected by image acquisition device. The obtained image is close to Figure 5, then we can achieve the correct decoding of the pre-stretched QR Code. 


\section{Software Implementation and Summary}

According to the above principle, the method of design and identification of anti-distortion two-dimensional code on cylindrical surface based on pre-stretching can be achieved by software. Specific steps are as follows.

Firstly, open the normal QR Code which will be processed.

Secondly, obtain the length of the image and the length $(\mathrm{cm})$ of the cylinder carrier.

Thirdly, pre-stretch the normal QR Code and save the processed image. Then you can get the pre-stretching QR Code picture as shown in Figure 7.

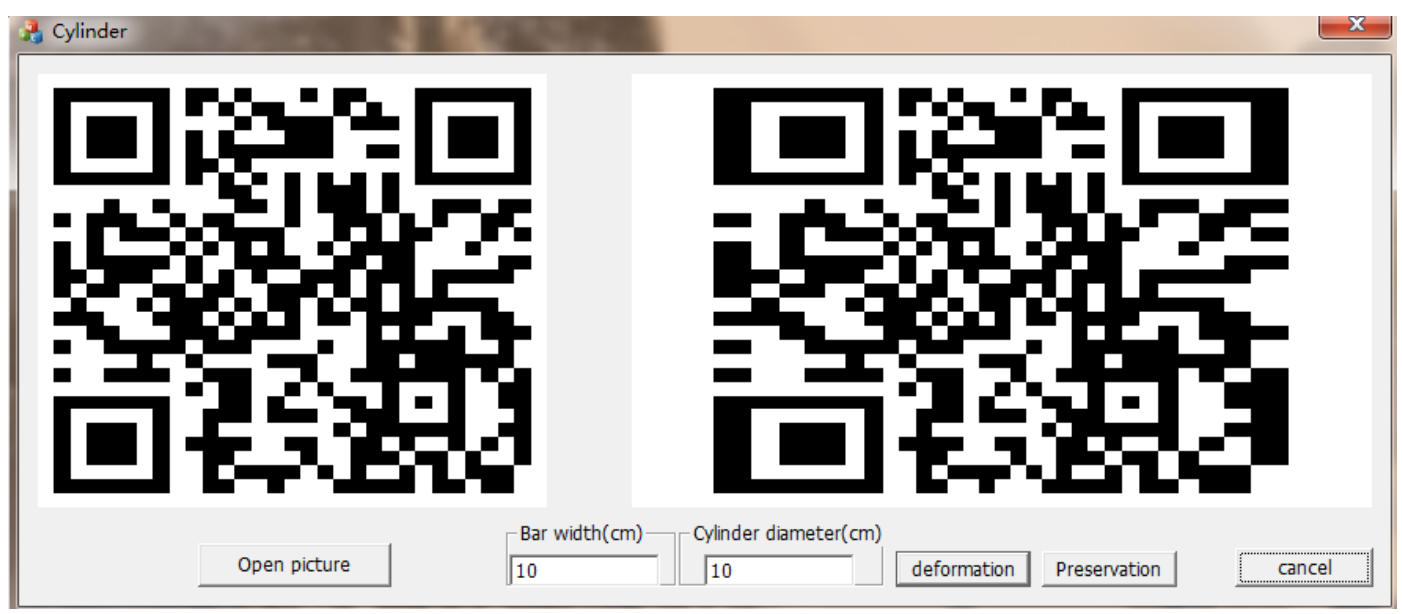

Figure 8. Partial Perspective of a Cylindrical Carrier

We assume that the diameter of the cylinder is $10 \mathrm{~cm}$, and the width and height of the normal QR Code is $10 \mathrm{~cm}$ as shown in Figure 5. We print this image on the surface of cylinder, then we can get the following image through the image acquisition device as shown in Figure 9.

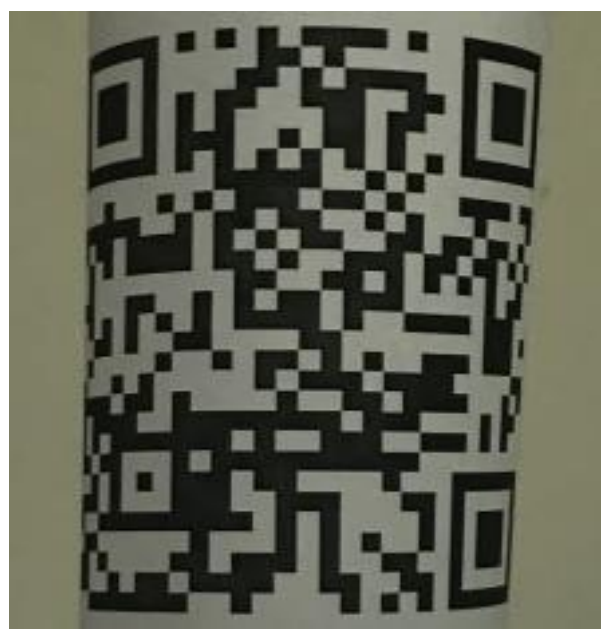

Figure 9. The Normal QR Code on the Surface of Cylinder

We get the pre-stretching QR Code by the above algorithm as shown in Figure 7. This picture is printed on the surface of a cylinder. Getting the QR Code image through the image acquisition device and then we can get the following image shown in Figure 10. 


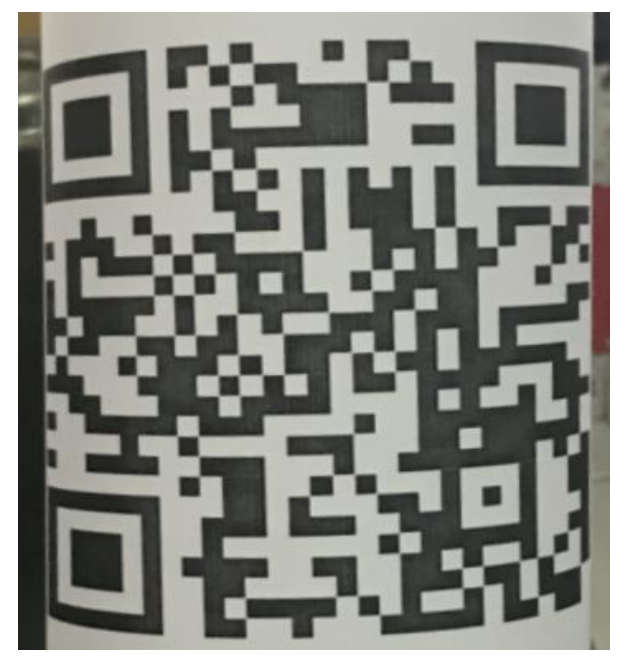

\section{Figure10. The Pre-stretching QR Code on the Surface of Cylinder}

In the end, the above image is identified by the decoding software and we can find out the following: When the normal QR Code print is printed on the surface of cylinder, the decoding device failed to identify image because the image is impressed. The pre-stretching QR Code can counteract the deformation of cylinder surface, so the decoding device can successfully identify image. This method of producing pre-stretching QR Code can increase the application occasions of QR Code.

\section{Fund Project}

1. Institute Level Key Project Funded by Beijing Institute of Graphic Communication(Ea201506).

2. Project of National Scientific Found (No.61370188).

3. Project of Beijing Municipal College Improvement Plan (PXM2017_014223_000063).

\section{References}

[1] W. Yi, "Application of Two - dimensional Code Technology and Introduction of Standardization”, China Standardization, vol. 5, (2006), pp.25-26.

[2] RUSSA, "Two dimensional bar codes", Adams Communications, vol. 27, no. 4, (2002), pp. 15-18.

[3] Z. Z. Jian, "Research and Implementation of QR Two - dimensional Code Coding and Decoding Technology", Cheng Du: University of Electronic Science and Technology, (2010).

[4] N. Gammer, T. Cherrett and C. Gutteridge, "Disseminating real-time bus arrival information via QR code tagged bus stops: a case study of user take-up and reaction in Southampton, UK”, Journal of Transport Geography, vol. 34, no. 2, (2014), pp. 254-261.

[5] G. Feng, "The Design and Implementation of Two-dimensional Barcode Recognition system on Mobile Phone", Beijing: Beijing University of Posts and Telecommunications, (2008).

[6] G. S. Spagnolo, L. Cozzella and M. D. Santis, "New 2D barcode solution based on computer generated holograms: Holographic barcode", //Communications Control and Signal Processing (ISCCSP), 2012 5th International Symposium on. IEEE, (2012), pp. 1-5.

[7] A. L. Talapov, "Ground State, Spectrum, and Phase Diagram of Two-Dimensional Incommensurate Crystals", Physical Review Letters, vol. 42, no. 42, (2015), pp. 65-67.

[8] L. N. Zhong and Y. J. Yu, "Recognition of Two-dimensional Bar Code Based on Fourier Transform", Journal of Image and Graphics, vol. 8, no. 8, (2003), pp. 877-882.

[9] G. V. Alekseev, "Control of boundary impedance in two-dimensional material-body cloaking by the wave flow method", Zh.vychisl.mat.mat.fiz, vol. 27, no. 4, (2013), pp. 2044-2016.

[10] X. P. Li and B. H. Yan, "Two - dimensional code overview and application", Screen printing industry, vol. $8, \mathbf{( 2 0 1 3 )}$, pp. 52-55. 